\title{
Design of Portable Color Blindness Aid System Based on Camera*
}

\author{
Qihua Chen ${ }^{1}$ \\ 1. School of Information Engineering \\ Wuhan University of Technology \\ Wuhan, China \\ Email:1903489432@qq.com
}

\author{
Dashun Que ${ }^{1,2}$ \\ 2. Key Laboratory of Fiber Optic Sensing Technology and \\ Information Processing, Ministry of Education \\ Wuhan University of Technology \\ Wuhan, China \\ Email:dsque@sina.com
}

\begin{abstract}
Combining with video acquisition, signal processing, single chip microcomputer, wireless communication and other multiple technologies, we design a portable color blindness aid system based on camera. We have completed the overall design of the system and the design of hardware and software. Using MSP430 single chip microcomputer, OV7670 camera module, HC-05 Bluetooth module and ISD4004 voice module to make the system more intelligent. Experimental test results show that the system can realize the function of color identification and broadcast of scale as well as voice. Compared to the traditional color recognition system, it has the advantages of identifying more kinds of colors, identifying higher precision, identifying greater distance etc, and it also has the characteristics of low power consumption and easy to use.
\end{abstract}

Keywords-Color blindness aid; Color identification; Camera; MSP430; ISD4004

\section{INTRODUCTION}

Color blindness patients are always the vulnerable groups of the society, whether partial color blindness or achromatopsia, shortage of color identification ability, having a great impact on all aspects of their lives. At present, the traditional color recognition system is mostly done based on color sensor, mainly using TCS230. Because of using the filter method to get the component of RGB, color recognition category is very limited by using the color recognition system of this color sensor, a dozen has already a very good effect, and the recognition accuracy is not particularly high, at the same time, identification module to restart, replacing the light source are in need of white balance adjustment in such situation, so the identification accuracy is not good because of some human error. The optimal distance detected by color sensor TCS 230 only have $10 \mathrm{~mm}$, it will bring great inconvenience by using such color recognition device. At the same time, the recognition effect is disturbed seriously by light factors of outside ${ }^{[1]}$. Therefore, it is not good helpful for color blindness.

The typoscope of color blindness designed in this paper will overcome the shortcomings of traditional color identification device. It is a kind of portable device, which can recognize object colors efficiently, then delivery to the color blindness through the scale and voice. It combines video

\footnotetext{
* Fund project: 2014 "national college students' innovative entrepreneurial
} training plan" project (20141049709005). acquisition, signal processing, single chip computer, wireless communication and many other technologies to achieve assisting to color blindness effectively.

\section{OVERALL DESIGN OF THE SYSTEM}

The vast majority of monochromatic light can be decomposed into three colors including red, green and blue. This is the most basic principle of colorimetry, which is call trichromatic theory. Other colors can be determined by these three kinds of color in different proportions of synthesis. In the RGB color model, as shown in Fig. 1, 3 axis of the cube are $\mathrm{R}, \mathrm{G}$ and $\mathrm{B}$. The origin corresponds to black $(0,0,0)$, the most distant from the origin of the vertex corresponds to white $(255,255,255)$, any color has a unique RGB value to correspond it ${ }^{[2]}$. This system mainly based on this principle to complete color recognition through extracting component of each color by the camera and single chip microcomputer.

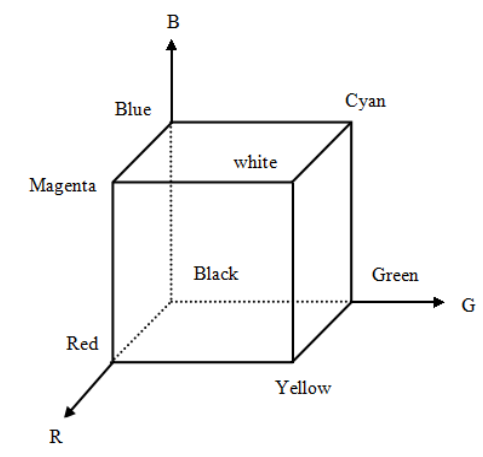

Fig. 1. RGB Color Model

This color blindness visual aid system mainly consists of video capture module, wireless communication module, single chip CPU, output module of scale and audio. Video capture module collects the object color information, then transfer to the single chip microcomputer processing center through the Bluetooth wireless transmission module. The MCU will convert the color signal received scale and audio signal, and control the output of scale and audio module, which can help patients with color blindness to identify colors. The block diagram of the overall system is as shown in Fig.2. 


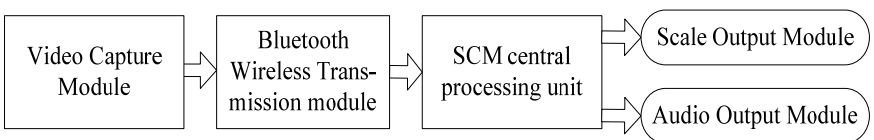

Fig. 2. Overall diagram of system

\section{SYSTEM HARDWARE DESIGN}

\section{A. Video Capture Module}

OV7670 CMOS camera with RGB output format, which is a new type of image sensor, produced by Ominivision Company, has the obvious characteristics of small volume and low operating voltage. All image processing function process including white balance, saturation, chroma, gamma curve and so on can all be programmed via the SCCB interface ${ }^{[3]}$.

Video capture module in this paper is mainly composed of OV7670 camera and AL422 chip, the hardware block diagram as shown in Fig.3. After OV7670 initialization, in the situation of opening field interruption and line interruption, the camera will parallel output one image data with 8 bits immediately into the buffer of AL422 when it comes a pixel interrupt every time. AL422 is a FIFO with the function of first in and first out, its memory capacity is $384 \mathrm{k}^{*} 8 \mathrm{bits}$ which can fully meet the requirement of database storage, it will transfer the data immediately using the Bluetooth wireless when filled with data of a filed.

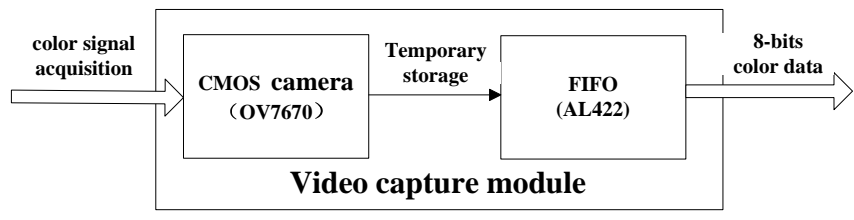

Fig. 3. Video capture module diagram

\section{B. Central Processing Module}

MSP430 Series MCU is a 16-bits MCU with ultra low power dissipation which is marketed by TI since 1996. It has a mixed signal processor RISC, strong processing ability, fast computational speed, ultra low power, and it has the advantages of convenience and efficient development environment.

In this paper, the control core of the color recognition system is the minimum system composed mainly by MSP430F149 chip that produced by America TI company and corresponding interface modules. The design of the MSP430 minimum system is simple, using external crystal oscillator of $8 \mathrm{MHz}$ and $32.768 \mathrm{KHz}$, reset circuit designed into button reset and automatic power on reset ${ }^{[4]}$.

\section{Communication Module}

This paper use a master-slave integrated Bluetooth serial module HC-05. This module is very small, only $16 \mathrm{~mm} * 32 \mathrm{~mm}$, connected with external by $6 \mathrm{pcs}$ pins with $2.54 \mathrm{~mm}$ spacing, in support of a very wide range of baud rate: $4800-1382400$, and the module is compatible with $5 \mathrm{~V}$ or $3.3 \mathrm{~V}$ single chip system, can be conveniently connected with various sensors and the single chip microcomputer, the use is very flexible. Schematic diagram is as shown in Fig. 4.

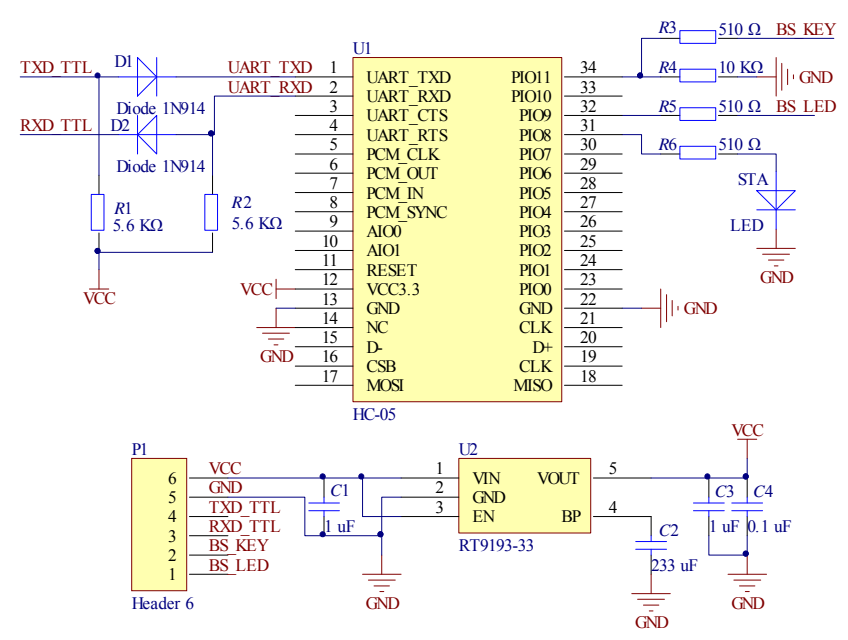

Fig. 4. HC-05 Bluetooth module principle diagram

\section{Scale and Audio Output Module}

Scale output module uses MSP430F149 single chip microcomputer to output square wave of different frequencies, then amplify it through the LM386 power amplifier circuit. Because of different colors correspond to different colors frequencies, different color frequencies corresponding to different voice frequencies, different voice frequencies corresponding to different scales, thus the corresponding color signal can be showed through the scale.

Audio output uses ISD4004 voice module, mainly consists of ISD4004 voice recording chip and LM386 power amplifier system. First of all, recording related color voice information at different address space of voice module through the serial port, then it can play the corresponding color voice by calling its address ${ }^{[5]}$. The module principle diagram is as shown in Fig.5.

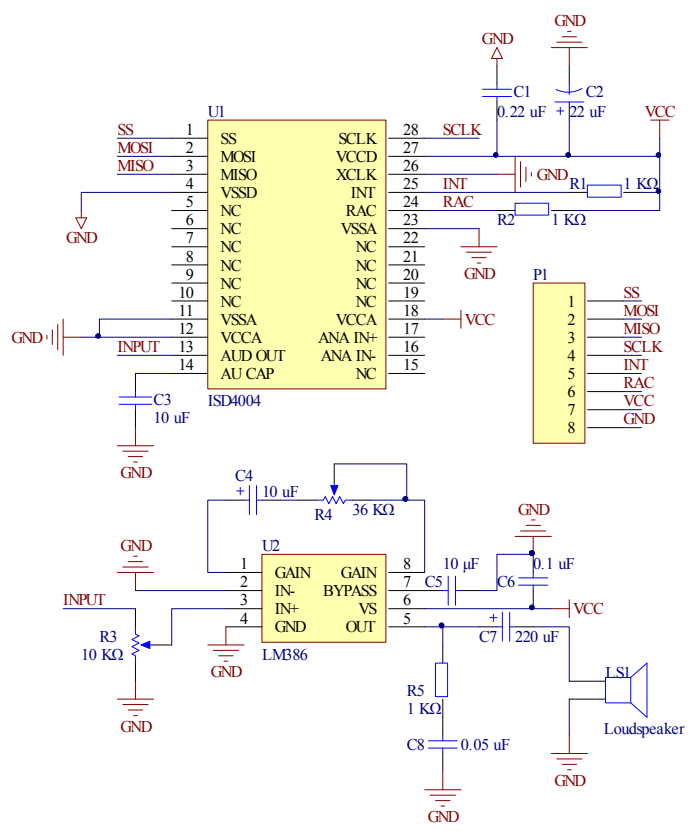

Fig. 5. Circuit principle diagram of audio output module 


\section{SYSTEM SOFTWARE DESIGN}

\section{A. Overall Design of the System Software}

The system software use the IAR software as debugging tools, $\mathrm{C}$ language as the software design language, the total design shows in Fig.6.

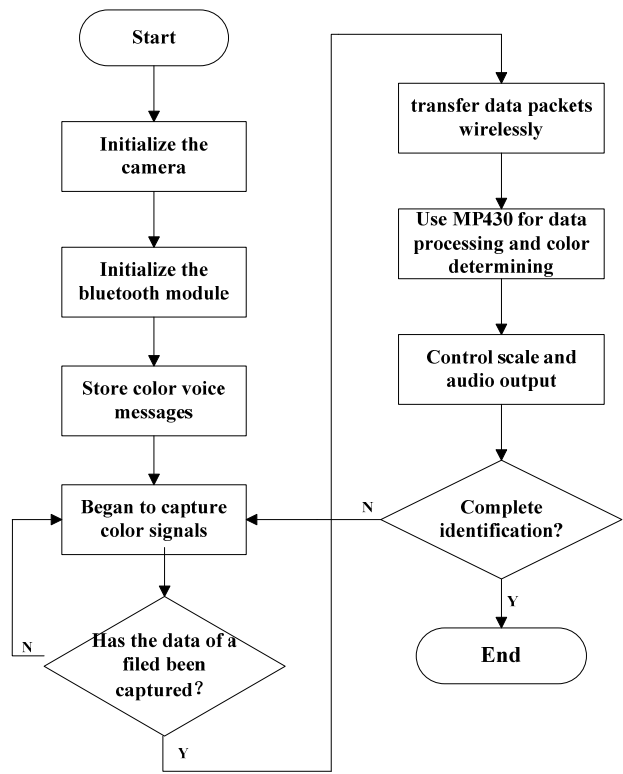

Fig. 6. Overall design of the system software

First initialize the OV7670 Camera, set the camera's data output format, contrast, exposure, white balance and other relevant parameters. Then initialize the Bluetooth module, at the same time entering color voice information to ISD4004. Then open OV7670 camera interrupt, start to capture color signals, when data capture completed, transport the data to the central processing center MSP430 through the Bluetooth wireless transmission. Single chip microcomputer analysis the color signal data and deal with it, complete the color recognition, finally control scale and audio module to output.

\section{B. MSP430 MCU Signal Processing Software Design}

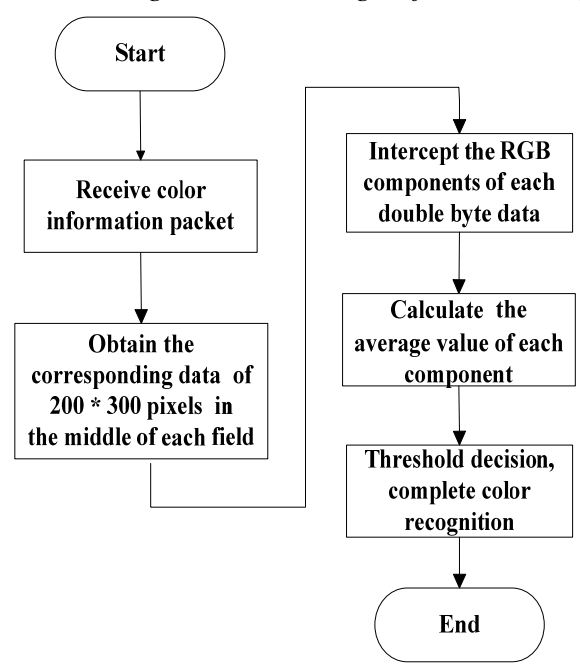

Fig. 7. MSP430 MCU signal processing software design
Set the OV7670 to QVGA format (240*320 resolution), taking into account the presence of the camera vanishing area, after the camera captures the image data and send to the MSP430 single chip microcomputer, the MCU dealt with the corresponding data of $200 * 300$ pixels in the middle of every field. Then establish an array of color information, intercept RGB component, calculate the average value, and compare with the RGB threshold to complete the color recognition. MSP430 MCU signal processing software design shows in Fig.7.

\section{System TeSt RESUlts AND ANALYsis}

First experiments to recognize these seven colors: red, green, blue, yellow, magenta, cyan, white. Physical map is as shown in Fig.8.

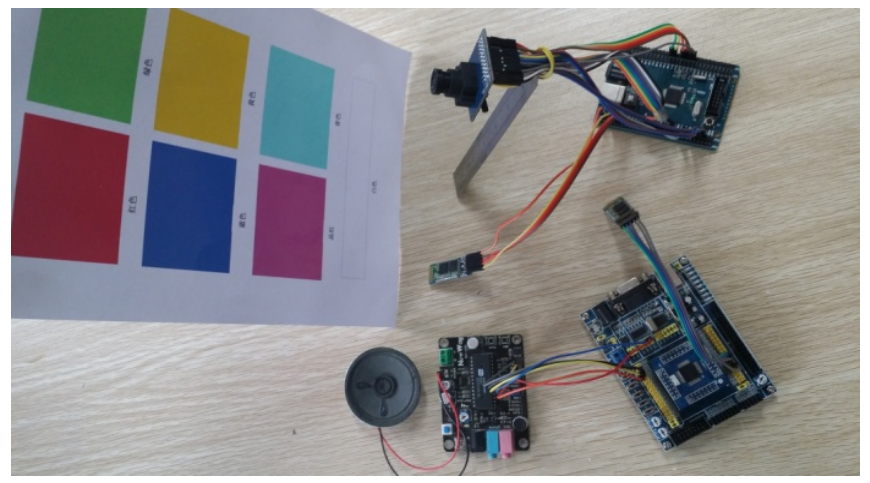

Fig. 8. Physical image of the whole system

OV7670 camera output the double-byte data in RGB565 format, after the algorithm processing of MSP430, R takes the higher five bits of the first byte, and $G$ takes the lower three bits of the first byte, and the higher three bits of the second byte, and B takes the lower five bits of the second byte. Each color is measured about 120 times, the average value of the corresponding RGB components as the test show in Table 1, according to the RGB components info of this table, it is possible to obtain the threshold value when judge the color.

TABLE I. AVERAGE VALUE OF SEVEN COLORS' RGB COMPONENT

\begin{tabular}{cccc}
\hline Color & R Value & G Value & B Value \\
\hline Red & 27 & 17 & 11 \\
Green & 10 & 37 & 10 \\
Blue & 11 & 21 & 22 \\
Yellow & 19 & 35 & 6 \\
Magenta & 20 & 21 & 19 \\
Cyan & 9 & 34 & 21 \\
White & 20 & 34 & 20 \\
\hline
\end{tabular}

When judging the color: $\mathrm{R}$ threshold is $15, \mathrm{G}$ threshold is 28 and $B$ threshold is 15. System test results under incandescent light conditions and under natural conditions are shown in Table 2. Through these test results, the system of color identification designed by this paper have a high accuracy, and it's recognition effect has almost none 
difference under incandescent light condition and natural light condition.

TABLE II. SEVEN COLORS TEST RESULTS

\begin{tabular}{cccc}
\hline Color & $\begin{array}{c}\text { Incandescent } \\
\text { light } \\
\text { (Success/All) }\end{array}$ & $\begin{array}{c}\text { Natural } \\
\text { light } \\
\text { (Success/All) }\end{array}$ & $\begin{array}{c}\text { Recognition rate } \\
\text { (Incandescent } \\
\text { /Natural) }\end{array}$ \\
\hline Red & $35 / 35$ & $35 / 35$ & $100 \% / 100 \%$ \\
Green & $34 / 35$ & $35 / 35$ & $97 \% / 100 \%$ \\
Blue & $35 / 35$ & $34 / 35$ & $100 \% / 97 \%$ \\
Yellow & $34 / 35$ & $33 / 35$ & $97 \% / 94 \%$ \\
Magenta & $33 / 35$ & $32 / 35$ & $94 \% / 91 \%$ \\
Cyan & $31 / 35$ & $34 / 35$ & $89 \% / 97 \%$ \\
White & $34 / 35$ & $33 / 35$ & $97 \% / 94 \%$ \\
\hline
\end{tabular}

According to certain color threshold, the system continues to test black, purple, orange, dark green, pink, gray and other colors, the recognition results are good. Finally after test, the system can recognize more than 20 colors. If we use the more accurate camera, it can recognize more colors.

From experimental test results, the typoscope of color blindness designed by this paper can overcome the shortcomings of traditional color recognition system. Compared to the traditional color recognition system using filtering method to extract the RGB components results, the system uses a camera and a single chip microcomputer to intercept the RGB components directly, which can recognize more types of color with higher accuracy and precision, and this design have the functions to adjust white balance, brightness, contrast automatically, and it is hardly affected by environmental factors. It can detect the maximum distance up to $8 \mathrm{~cm}$, higher more than $10 \mathrm{~mm}$ detected by traditional color recognition system, it can help the color blindness more easily and effectively.

\section{CONCLUSIONS}

This paper introduces a portable typoscope of color blindness, including its hardware and software design principle, function analysis and system testing. The system has the function of color recognition and broadcast of scale and audio and so on, compared with the traditional color recognition system composed by the color sensor, it has the advantages of identification of various kinds of colors, high identification accuracy, recognition distance and so on, and has the characteristic of lightweight and easy to carry. It provides a new assistive device for the achromate, which can enrich their spiritual world.

\section{ACKNOWLEDGMENT}

This research was supported by the 2014 "national college students' innovative entrepreneurial training plan" project (20141049709005) and was guided by professor Que, all support is gratefully acknowledged.

\section{REFERENCES}

[1] Ruiting Jiang. Weapon of Recognize Color - Color Recognition System [J]. Beijing: Electronic Production, 2011, 9 (1): 33-35, 58.

[2] Xiaoxuan Qi, Jianwei Ji. An Approach of Color Feature Evaluation in Color[C]//2011 Eighth International Conference on Fuzzy Systems and Knowledge Discovery (FSKD), 2011

[3] Deming Li, Jian Han, Guoqiang Jiang. Image Acquisition and Display System Design Based on OV7670 [J]. Beijing: Chinese Journal of Scientific Instrument, 2010, 37 (8): 30-33.

[4] Chao Wang, Cuicui Huang. The Minimum System Design and its Application Based on Single Chip Microcomputer MSP430F149 [J]. Guangdong: Modern Construction, 2012, 11 (1):28-30.

[5] Wenyi Li, Yanhe Shen, Xianzhao Lv. ISD4000 Series Voice Chip Development Platform Design[J]. Anhui: Journal of Suzhou University, 2012, 5(27):36-38. 